\title{
Modularization in material flow simulation for managing production releases in remanufacturing
}

\author{
Lorenzo Gaspari ${ }^{1}$ (D) Lorenzo Colucci $^{1}$ • \\ Steffen Butzer ${ }^{2} \cdot$ Marcello Colledani $^{1} \cdot$ Rolf Steinhilper $^{2}$
}

Received: 10 April 2017 / Accepted: 21 July 2017 / Published online: 4 October 2017

(C) Springer Science+Business Media B.V. 2017

\begin{abstract}
Remanufacturing is recognized as a major circular economy option to recover and upgrade functions from post-use products. However, the inefficiencies associated with operations, mainly due to the uncertainty and variability of material flows and product conditions, undermine the growth of remanufacturing. With the objective of supporting the design and management of more proficient and robust remanufacturing processes, this paper proposes a generic and reconfigurable simulation model of remanufacturing systems. The developed model relies upon a modular framework that enables the user to handle multiple process settings and production control policies, among which token-based policies. Customizable to the characteristics of the process under analysis, this model can support logistics performance evaluation of different production control policies, thus enabling the selection of the optimal policy in specific business contexts. The proposed model is applied to a real remanufacturing environment in order to validate and demonstrate its applicability and benefits in the industrial settings.
\end{abstract}

Keywords Remanufacturing $\cdot$ Kanban $\cdot$ Modularity $\cdot$ Simulation $\cdot$ Production control policies

\section{Introduction, motivation and objectives}

The increasing world population is leading to an upward momentum for resources demand, in order to satisfy emerging consumer requirements in the global market. However, this macrotrend poses a challenge on the need to decouple resource consumption and production to support a sustainable development. In this context, Circular Economy has been recently

Lorenzo Gaspari

lorenzo1.gaspari@gmail.com

1 Politecnico di Milano, Mechanical Engineering Department, via La Masa 1, 20156 Milan, Italy

2 Chair Manufacturing and Remanufacturing Technology, Universität Bayreuth, c/o LUP,

Universitätsstraße 9, 95447 Bayreuth, Germany 
proposed as a new paradigm for sustainable development, showing potentials to generate new business opportunities in worldwide economies, to increase a long-term competitive advantage [1] and to significantly increment resource efficiency in manufacturing. The application of closed-loop business models may enable to exploit materials potentials within multiple cycles, reducing emissions, energy requirement and resource consumption, ultimately preserving the welfare of next generations.

Focusing on the operational perspective of circular economy, remanufacturing is acknowledged among the most beneficial end-of-life product regeneration strategies. Indeed, it provides the possibility of preserving post-use products or components functions, regenerating them to their as-good-as-new conditions [2]. Although remanufacturing is gaining interest due to its profitability and environmental benefits, the related instability, uncertainties and complexity [3], particularly at an operational level, undermines its growth.

With the objective of providing increased robustness to remanufacturing processes while limiting inventory levels, this work proposes an innovative simulation framework for predicting the performance of remanufacturing systems operating under various production control policies within a digital environment, before the implementation in the real system. The proposed tool provides remanufacturing business stakeholders with an effective solution which supports the management of remanufacturing systems under evolving production targets, thus lowering the exposition of companies to input disturbances and uncertainties. Such objectives are pursued through the proposition of a generic and reconfigurable simulation model, which exploits a modular approach. The characteristics of each process module are customized in order to capture the features of typical remanufacturing processes, including disassembly, inspection, cleaning, regeneration, functional testing and re-assembly. Moreover, in the proposed simulation environment, the ability to compose different remanufacturing system architectures is provided, while maintaining an adequate level of detail to capture the main dynamics characterizing the system's behaviour. The model is also enriched with the capability of handling different production control policies. The ultimate aim of this work is to support the design, reconfiguration and management of robust remanufacturing systems, adaptable to variable production targets and input post-use product flows and conditions.

The remainder of this paper is structured as follows. In the next section, a literature review on remanufacturing planning and control methods is provided, also highlighting the existing gaps and limitations. In section 3, the scientific approach proposed in this paper is outlined and the detailed description of the simulation model is proposed. Numerical validations through comparison with existing performance evaluation methods, targeted to a sub-set of low complexity system configurations, are also provided. In section 4 , the application to a real remanufacturing industrial case is demonstrated, and potential benefits are discussed. In section 5, the main conclusions are drawn.

\section{Literature review}

Since decades, simulation is a well-known approach for business forecasts and development, mainly applied to the manufacturing domain. In-spite of the complexity of remanufacturing and the related uncertainties and disturbances, relatively little effort has been devoted to the application of simulation in remanufacturing systems. Despite the presence of studies rooted in the nineties [4] [5], simulation has always faced limitations in remanufacturing applications. The main reason is that the proposed simulation models 
were circumscribed to specific, case-dependent assumptions, which imply difficulties in the adaptation and generalization of the presented solutions over different remanufacturing scenarios. Among these works, Souza and Ketzenberg modelled the operations occurring in remanufacturing activities [6] and focused their efforts on determining the optimal longrun product mix, maximizing profit subject to a service time constraint [7]. Moreover, Zhang, Ong and Nee tackled the problem of process planning and scheduling through the application of a simulation-based framework, optimized by a genetic algorithm [8].

The concept of modularity has been largely deployed in the field of manufacturing, both at product and production system design level, and its application provided significant benefits in terms of manufacturing costs and lead times [9] [10]. In material flow simulation, modularity represents an effective way of reducing model development times. Smith and Valenzuela considered the development of modular templates to ease, foster and accelerate the deployment of simulation in specific domains [11]. The authors asserted that significant complexities in modular models are hidden within the templates, enhancing an easy and fast implementation of the simulation. Using standard modules and formalized interfaces, a complex system can be modelled easily and effectively with an emergent approach, deploying modules selection and integration. However, the complexity in capturing the important characteristics of the process modules and defining the right level of details have resulted in limited applications of modular simulation models in remanufacturing, privileging modelling approaches, dedicated to specific system settings. Martínez and Bedia developed process modules for the case of Just in Time (JIT) manufacturing processes [12]. They applied them to the modelling of a U-shaped line, in order to show the appositeness of the developed model. Lee and Choi designed a modular reconfigurable simulation-based framework for the creation of planning and scheduling systems within a manufacturing context [13]. Applications of modular simulation models to remanufacturing systems are not being developed so far. A topic that has barely been considered in material flow simulation models for remanufacturing systems, is the application of production control policies, meant to reduce the variability that remanufacturing plants are exposed to. The approaches in literature aim at finding a generic optimal policy or at looking in detail to the production release of a particular station, mainly the disassembly one. Some of these works are based on analytical models [14]. For example, Veerakamolmal and Gupta developed a procedure that sequences multiple and single-product batches through disassembly and retrieval operations to minimize machines idle time and make-span, determining lot sizes according to the number of arrivals per product in a given time period [15]. Moreover, Gupta and Al-Turki introduced the so-called Flexible Kanban System (FKS), which uses an algorithm to dynamically and systematically manipulate the number of Kanban in order to offset the blocking and starvation caused by uncertainties during a production cycle [16]. Korugan and Gupta suggested the application of a single-stage pull type control mechanism with adaptive Kanban and state-independent routing of demand information, for the management of a hybrid production system [17]. Furthermore, Tang and Teunter explored the economic lot-scheduling problem (ELSP) within the context of remanufacturing. Their challenge was to determine both the lot size and the sequence of production for each product minimizing the holding and set-up costs [14]. Other attempts are based on heuristic models. Among these, Teunter, Kasparis and Tang developed a mixed-integer program to solve the economic lot-scheduling problem with returns (ELSPR) when manufacturing and remanufacturing operations are performed on separate dedicated lines [18]. Additionally, the same authors deepened the study of the ELSPR by developing fast yet simple heuristics that can provide nearly optimal solutions [19]. Guide and Srivastava examined the use of safety 
stocks in a material requirements planning (MRP) production system and the impact of the location of buffer inventory on remanufacturing performance [20]. In a later work, Guide and Srivastava evaluated, through simulation, the performance of four order release strategies level, local load oriented, global load oriented, and batch - and two priority scheduling rules first-come-first-served (FCFS) and EDD - towards five performance criteria, over a real case [21]. Guide, Souza, and Van der Laan used simulation to prove results from an analytical model. They found that, under certain capacity and process restrictions, delaying a component to the shop after disassembly never improves the system's performance [22]. The provided results show margins of improvement thanks to the production control, nevertheless affected by the specificity of the context and external uncertainty.

Analysing in detail the simulated remanufacturing systems found in literature, it is visible how strictly connected they are either to a single case or to a specific industry. Moreover, the simulations aim at providing detailed assessment focusing on single stations, losing the attention over the entire system, or vice versa. In general, research could benefit from simulation models that - on the one hand - are general and easily adaptable to different industries and - on the other - are able to handle great performance granularity both at system and at station level.

As highlighted by this analysis, production control management in remanufacturing has never been tackled by flexible, modular and easily reconfigurable simulation models, thus undermining their effective design and implementation in industrial settings. To fill this gap, this paper proposes a new user-friendly simulation environment, which predicts the relevant performance measures of remanufacturing systems as a function of the applied production control policy. The suggested method paves the way to the design and management of robust remanufacturing systems that can better suit evolving target demands and post-use product conditions.

\section{The proposed simulation model}

The research has been methodologically approached following pre-defined steps for a simulation study [23], as reported in Fig. 1.

Due to the objective of having a generic simulation framework, the main difference concerns the data collection step. Hence, data are not recorded before implementing the model, rather throughout the setting phase. When system data are needed, user interfaces enable recording them and embed the flexibility for adapting the system according to the specified values.

\section{Model Conceptualization}

The whole model architecture relies upon a conceptual framework built with a Class Diagram in Unified Modeling Language (UML). Already rooted in such representation, is the modularity: it implies the development of System Modules, intended as self-independent packages of objects playing distinctive roles. Having a pre-defined modular framework implies three main benefits:

i. Starting the implementation from an already coded set of tasks and logics represents a saving of time.

ii. Modularity implies flexibility, which is fundamental to react in unstable scenarios as remanufacturing is.

iii. The programming complexity behind an entire system is divided into smaller problems among modules. 


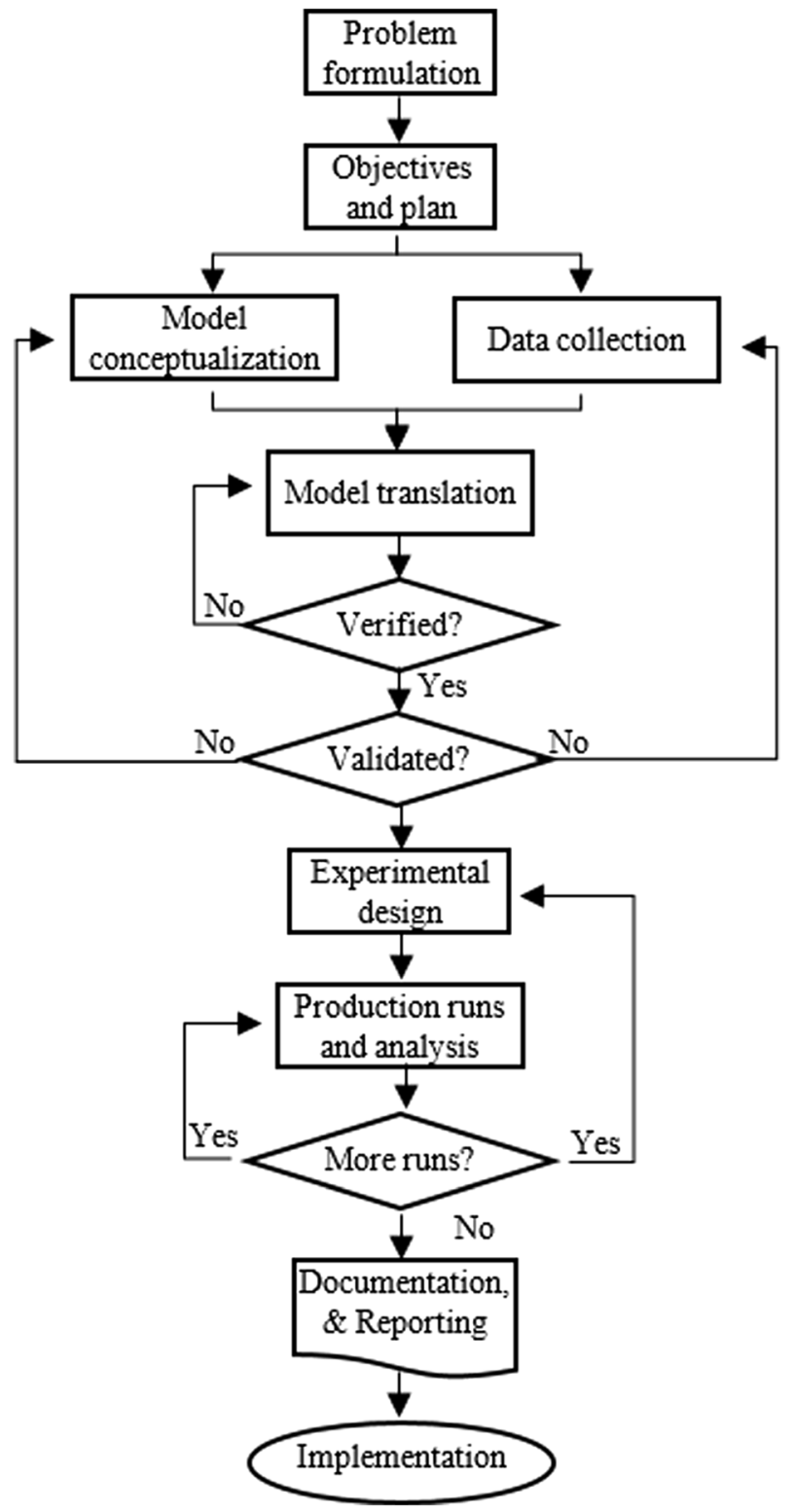

Fig. 1 Steps for a simulation study

The developed framework enables modelling the remanufacturing processes through a product-oriented architecture. Indeed, each System Module - which is deployed to build up the process representation - does not represent a physical resource, but its respective carried activity. It means that, if a resource is available in more than one technological path of cores, or target components, the respective System Module is replicated in the simulation framework as well. This perspective can be considered innovative compared to the process-orientated architecture, where there is an exact correspondence between physical resources and simulation-based representations. 


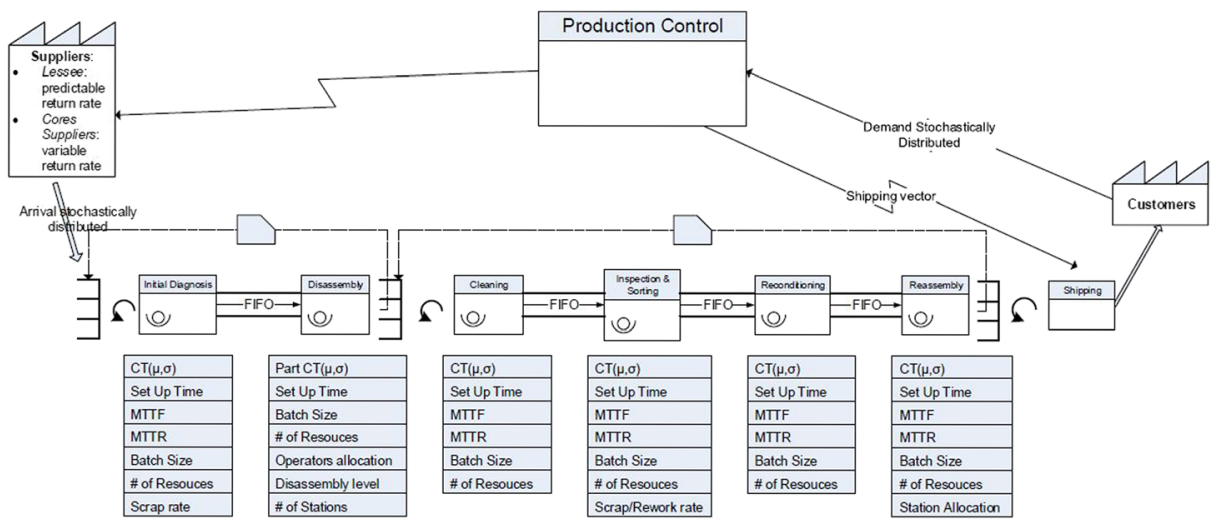

Fig. 2 Multi-Stage control, VSM representations

Each System Module is embedded with a Module_Kanban, a built-in frame, able to handle six different production control policies in two distinctive environments, namely Absence of Demand and Presence of Demand, which differ for controlling production taking into account - or not - the demand for finished goods [7]. Moreover, the six available policies refer to those described by Liberopoulos [24]. No WIP Control represents the full-speed-working configuration, in which cores are processed as soon as the needed resource is available. Flowline sets a control over the production through a blocking mechanism, by capping buffer sizes. In addition to these policies, other notorious Kanban-driven controls are taken into account, such as: Control at station level, ConWIP, Multi-Stage and Echelon (Figs. 2 and 3).

In the Control at Workstation level, the WIP amount is directly managed at every process stage, oppositely, in the ConWIP Control, the WIP is regulated at system level. In-between the last two shown policies, the Multi-Stage and Echelon control, can be reasonable trade-offs between their respective pros and cons. These configurations have a user-defined number of control points, however, in the last one, Kanban are not detached from the product before entering in the subsequent stage. For this reason, it leads to a global control over WIP and not a local one, as in the Multi-Stage case.

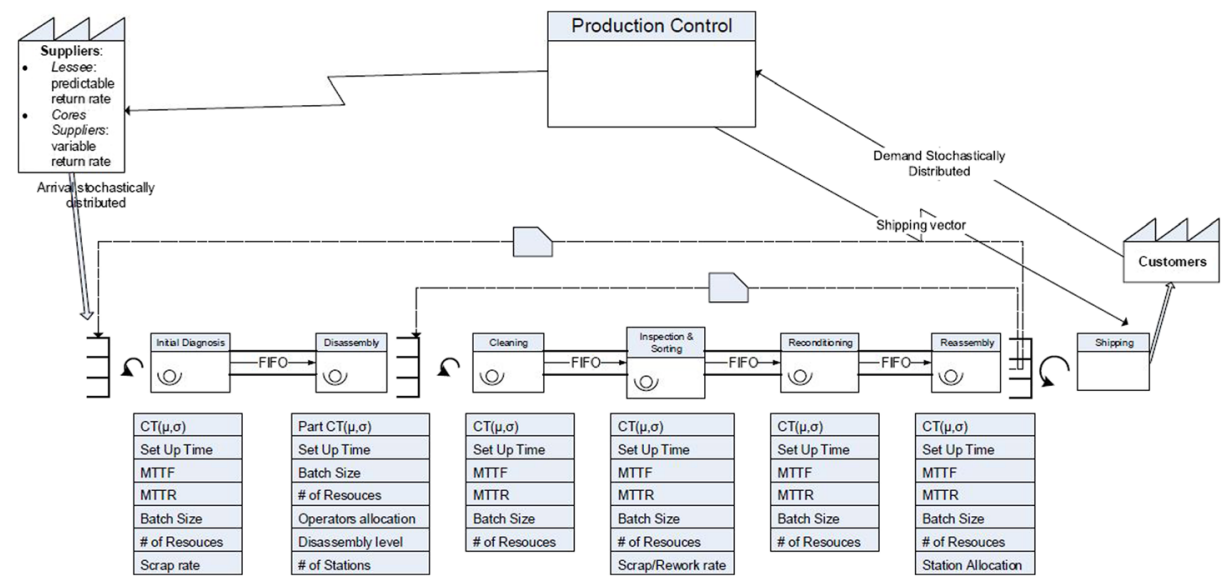

Fig. 3 Echelon control, VSM representation 


\section{Model Description}

As anticipated, the model hereby is a pre-defined framework, in which the remanufacturing process can be represented following a guided path where the user can specify the data of the analysed system. Throughout such procedure, the whole architecture is built, putting together the required System Modules. Within these Frames, the Module_Kanban regulates the behaviour of the material flowing across the related System Module by coordinating authorization cards, according to the rules of the chosen production control policy. The following paragraph focuses on the characteristics and functions associated with System Modules and Module_Kanban.

\section{System Modules and Functioning}

Starting from Steinhilper's work [25] and extending the identified processes related to remanufacturing for modelling reasons, the identified System Modules are: Sorting, Inspection, Cleaning, Reconditioning, Disassembly, Reassembly and Outsourcing (Fig 4).

Each System Module is conceptually made of two different parts: the former, with the socalled homologous objects, which are the same independently from the System Module in question, needed for interfacing each module with the system; the latter, which concerns objects and methods that are peculiar of the process associated.

The target architecture and the correct production mechanism are obtained leveraging on similar interfaces and adaptable controlling methods, which together constitute the homologous objects. Interfaces are developed exploiting Siemens Tecnomatix ${ }^{\circledR}$ specific library objects: they are characterized from common names or labels, and enable the communication with system-level controlling methods. The aforementioned adaptable methods are programmed through the so-called anonymous identifiers, which are coding elements able to point at specific objects or entities, without specifying their location in absolute terms, rather referring to the method they embed or they trigger.

The homologous objects, reported in Fig. 5, are in charge of:

- Connecting to other System Modules.

- Managing the selected production control policy, through Module_Kanban.

- Managing the resource queue, batch release and task execution.

- Updating M1 available capacity, before and after the task is performed.

- Modelling the transportation time from a buffer to the performer task.

- Requesting and setting of System Module input data, which are processing and set up time, amount of equal resources, handling and resource batch size, entry and exit time and set up conditions.

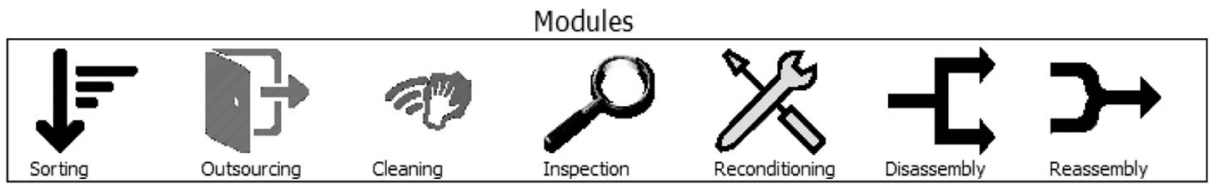

Fig. 4 System Modules 


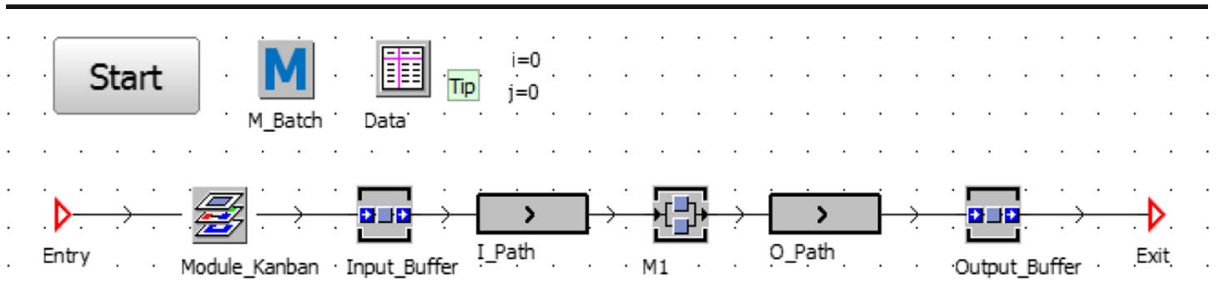

Fig. 5 System Module's interfaces

As mentioned, one part of the internal structure of System Modules changes in order to perform the specific set of activities they are in charge of. The following paragraphs point out the differences between them.

At system level, a wide set of key performance indicators monitors the logistic performances of the material flow in the simulation. The recorded KPIs are mainly drawn from Hopp and Spermann [26]. Particularly, three KPIs areas are encompassed: throughput rate, work in progress (WIP) and throughput time. Table. 1 reports how the implemented KPIs cover different hierarchical levels of the system. On top of them, the actual throughput and the resource utilization are recorded.

Sorting The first System Module under analysis, represented in Fig. 6, deals with the sorting phase; generally, this process has the main function of characterizing a product over certain factors of interest. Converted to programming functionalities, this module allows the user to define - during the setting stage - some attributes, the range of possible values and their occurrence percentages. Afterwards, during the simulation run, entities are given different attribute values, according to the defined occurrences.

One consequence of being labelled with a certain attribute value is that the product could be accepted, rejected or reworked according to a certain detail, such as the presence of a specific qualitative defect. The second consequence is the possibility of having a different processing time for one of the successive task, which depends on a product feature as well. An example could be the level of rust characterizing a core, inversely proportionated to its removal process time.

Inspection This System Module represents the process of accepting, discarding or defining a rework path for a certain product. During a remanufacturing process, this activity occurs several times throughout the process, in order to ensure the target quality level. The Inspection module can be used independently at different stages of the process (Fig 7).

In case the process entails a scrap rate higher than zero, and the part under analysis is an already disassembled component, the user has the possibility to re-order the component as new. In this case - with a delay that has to be defined - the component is addressed directly at its respective buffer prior to the reassembly stage. This possibility is given because an

Table 1 Model KPIs at system level

\begin{tabular}{llll}
\hline & Work in Progress & Throughput rate & Throughput time \\
\hline System level & Inventory Type & Th_Rate_Mean & Th_Time_Mean \\
& Avg Wip in Kanban & Th_Rate_StDev & Th_Time_StdDev \\
Efficiency & loop & UB_Rate & Time_0 \\
WS level & - & Th_Rate_Efficiency & Th_Time_Efficiency \\
\hline
\end{tabular}




\section{Sorting}

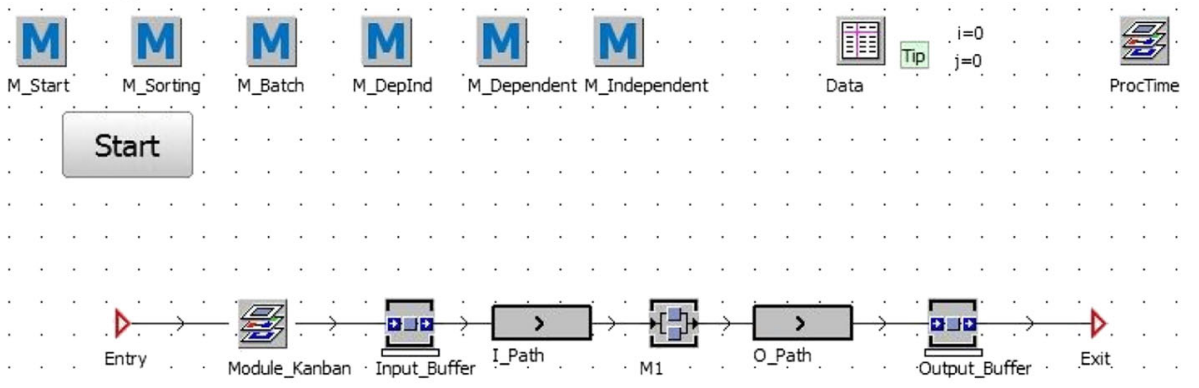

Fig. 6 Sorting Module

unbalanced scrap rate for different components could lead to an uneven storage level across them.

Cleaning and Reconditioning In this model, Cleaning and Reconditioning Modules are conceptually identical. This decision reflects the fact that, although the carried out process is different, when the target of the simulation is the evaluation of logistic performances, what matters of the model is the time delay occurring due to the represented activity. None of these two stages has features affecting the material flow behaviour, from the time needed for processing. For this reason, one System Module would be enough for representing both families of activities: despite that, two separate System Modules have been built for the sake of comprehensiveness (Figs. 8 and 9).

Outsourcing This module aims at representing those process phases which are performed externally to the company. It simulates the outsourcing time, that is the time components spend out of the system.

Unlike the other System Modules, this one does not contain all the homologous Objects pointed out in paragraph 3.2.1. The first reason is that there is no need for the production

\section{Inspection}

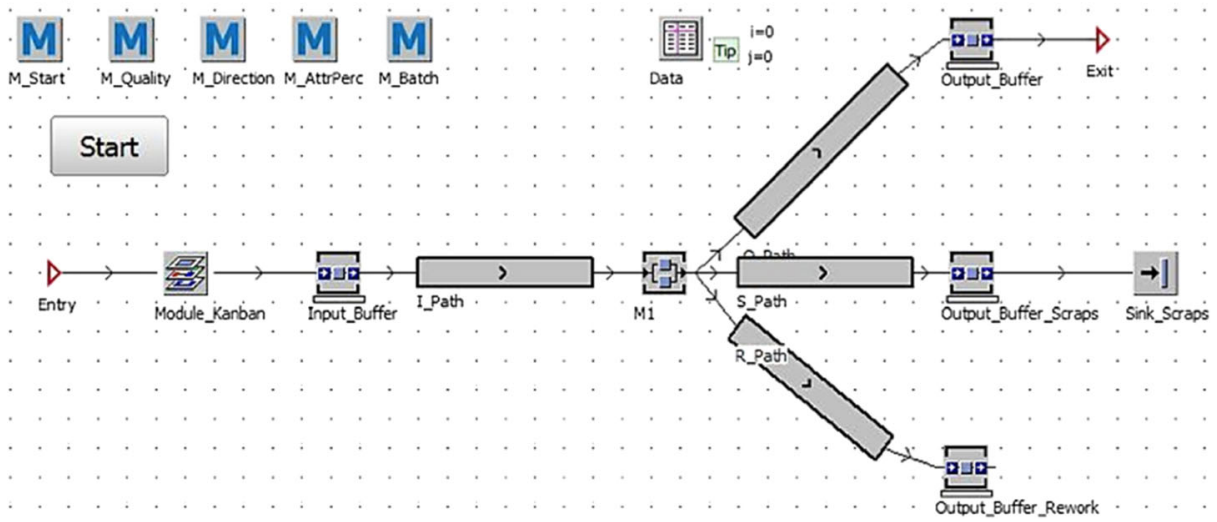

Fig. 7 Inspection Module 


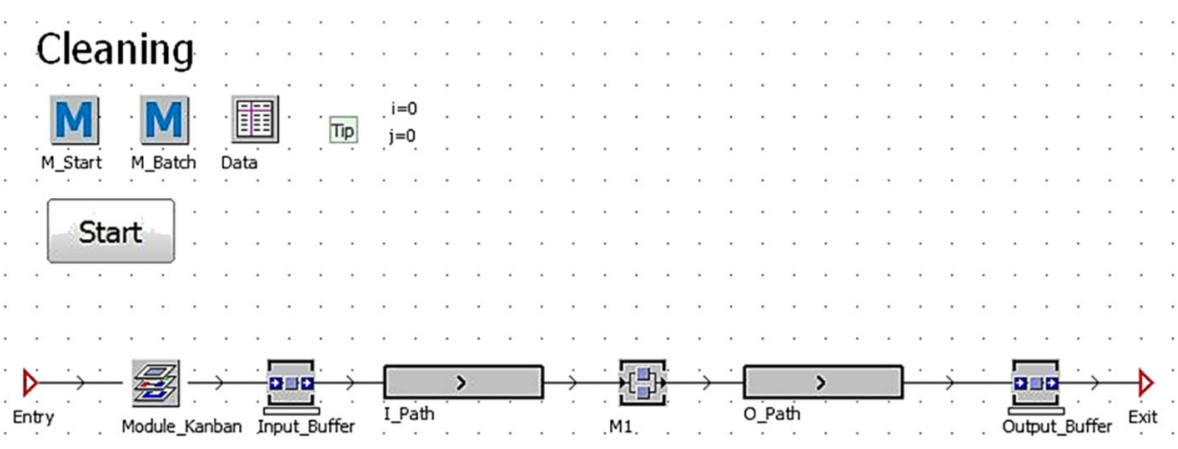

Fig. 8 Cleaning Module

policies management, such as Module_Kanban deployment. In fact, the products are sent out of the company boundaries, as it is not feasible to attach Kanban on them, or it would be incoherent to detach authorization cards since outsourced parts account for the overall WIP amount. Secondly, having as main interest the outsourcing time, only two Lines Internal_path and External_Path - have been included, simulating the logistic time needed to ship and receive the outsourced components batch. Additionally, the user has the possibility to consider more than one outsourcer for the same component, with different times and batches characteristics (Fig 10).

Disassembly The Disassembly is the System Module in which the decomposition of the Core into its Components occurs. This process is modelled with more details because the decisions taken at this stage - e.g. Target Components - affect the entire remanufacturing process (Fig 11).

The functioning of the module is structured in order to assure the user a high flexibility in simulating different disassembly scenarios, enabling sensitivity analysis of the system under the variation of one or more of its input data. This flexibility is aligned with the generality and comprehensiveness of the required data as input of the module, in order to cover a broad range of disassembly configurations, giving the user multiple levers of intervention for a deep understanding of the operations. Overall, in addition to the aforementioned data needed for setting up a resource, this stage requires the user to define:

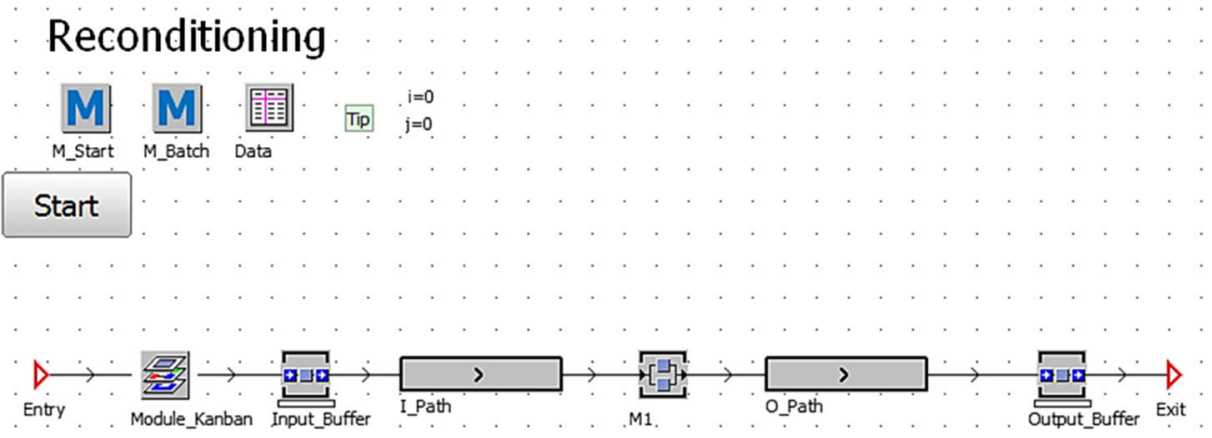

Fig. 9 Reconditioning Module 


\section{Outsourcing}

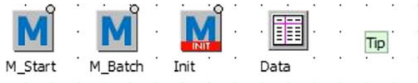

Start

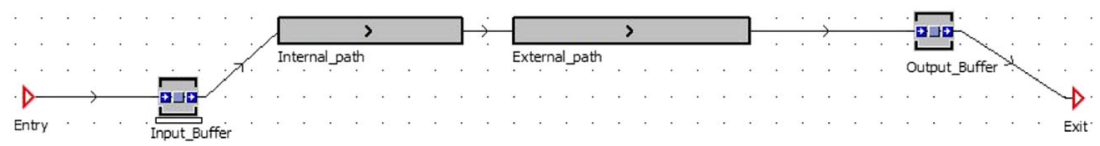

Fig. 10 Outsourcing Module

i. Disassembly level, determining the target components to be remanufactured

ii. Disassembly task sequence, with the related processing and setup times

iii. Disassembly line balancing, allocating tasks to workstations

Indeed, another relevant characteristic of the Disassembly is the presence of subframes Station, shown in Fig. 12, representing disassembly manual workstations. According to the line-balancing problem logics, the user can simulate the allocation of disassembly tasks over different stations. A further variable available to the user, concerning the workstations, is the amount of human resource and the respective allocation and sequence of tasks performed.

Finally, in this phase, it is possible to understand clearly the product-oriented architecture used for modelling the system. In fact, from this step on, the flow of Entities is divided because each target component follows its own technological routing until the reassembly stage. For this reason, Disassembly provides an amount of output buffers corresponding to the number of components defined as target.
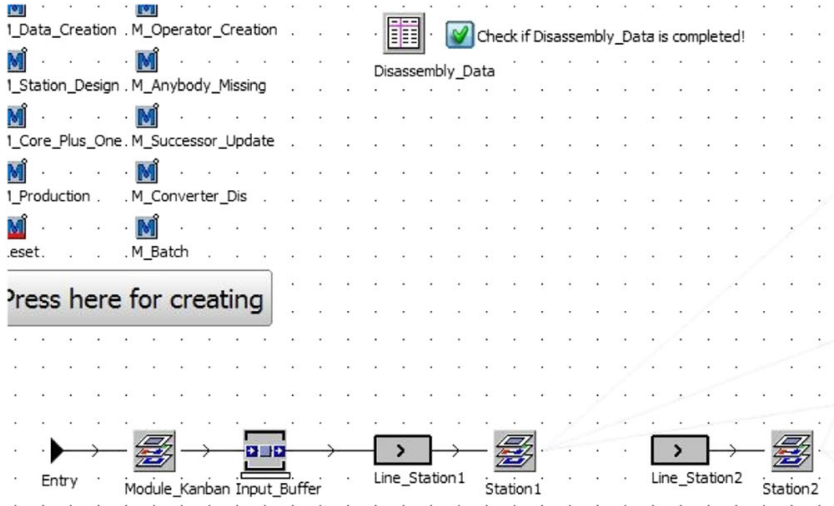
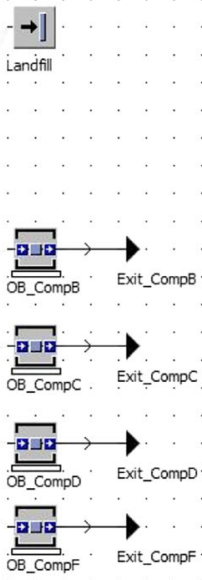

Fig. 11 Disassembly Module 


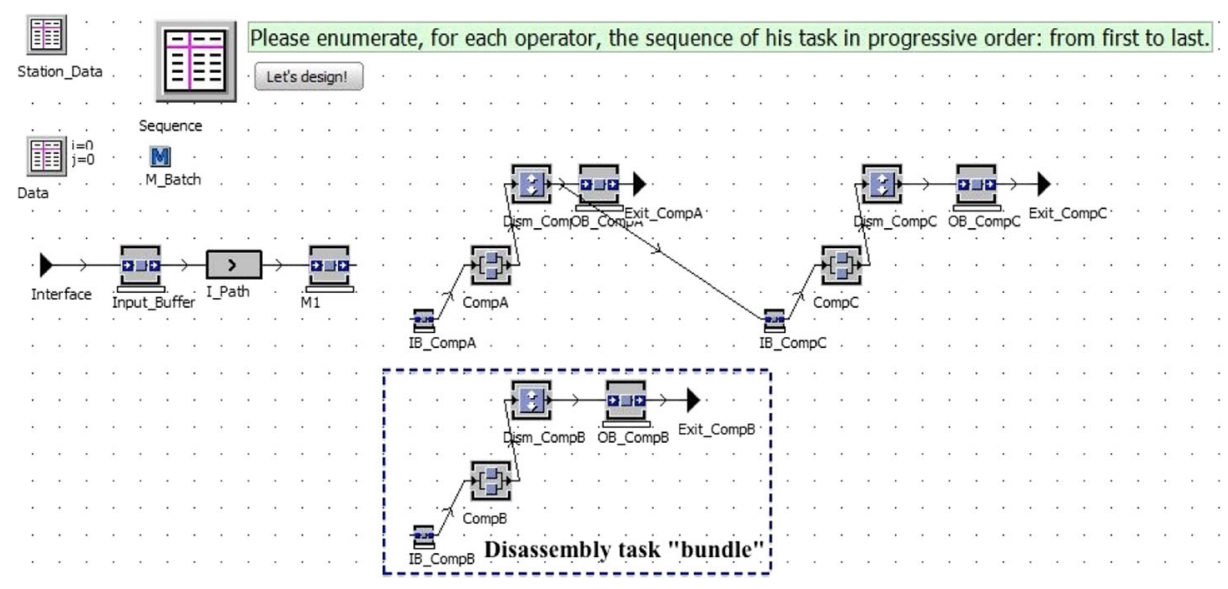

Fig. 12 Disassembly Station

Reassembly Differently from Disassembly, which has been modelled as a humanbased process, the Reassembly has been conceived considering the introduction of automatization. The range of reassembly configurations is wide. The module is structured in order to be able to represent at least the three main typologies of reassembly configurations:

- Fixed position, in case the product is assembled in a single site, rather than being moved through a set of assembly stations.

- Assembly line, meant as a fixed and unique path where the product is progressively assembled.

- Decoupled Assembly stations, where each phase of the assembly process of a product type is assigned to a specific station.

The three categories mentioned are not modeling configurations that the user can select as baseline layout. On the contrary, they are theoretical structures that could be achieved leveraging on the number of Stations and the batch release mechanism.4

The module is characterized by the convergence of target components' material flows, moving into the sequence of reassembly stations specified by the user. In analogy with Disassembly, the module is provided with the sub-frame Station, which is replicated as many times as specified and which is in charge of modelling the assembly of assigned components. Furthermore, the module is provided with the subframe New_Components that has the possibility to reorder those components that are designed as non-target during the disassembly phase (Figs. 13 and 14).

\section{Module_Kanban}

This sub-frame is placed at the entrance of every System Module, except for the Outsourcing. Once the user selects the production control policy and specifies what the control points are, 


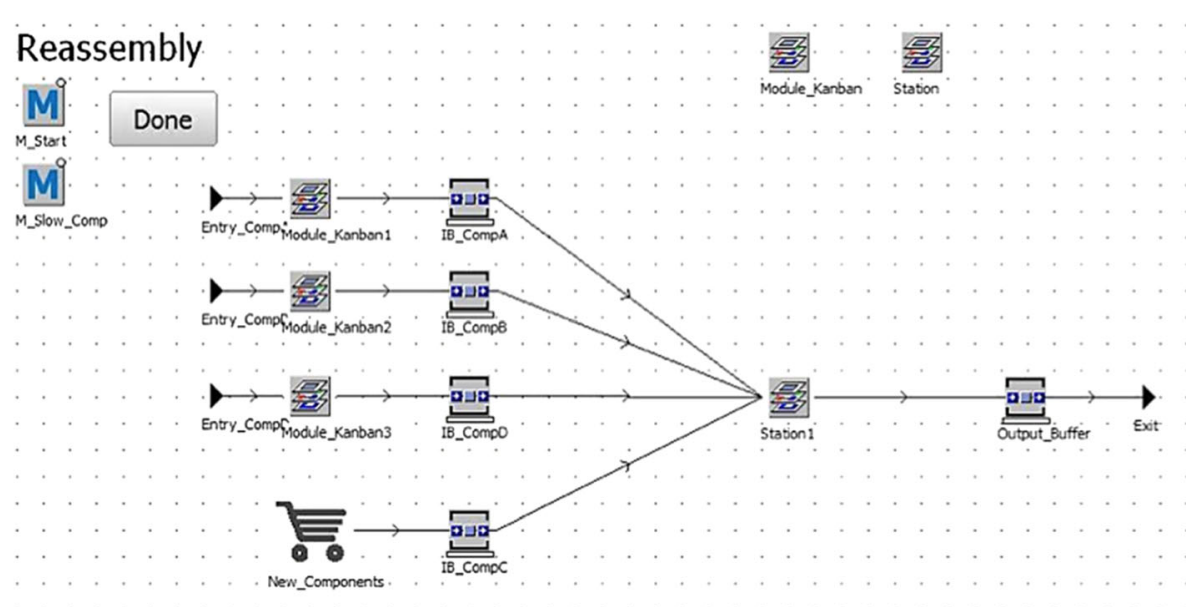

Fig. 13 Reassembly Module

deactivating them. If a Module_Kanban is deactivated, material flow simply crosses it without any effect. If activated, it represents the point in which material entities are bundled with a production - or demand - of Kanban anytime this is available (Fig. 15).

The implemented controlling methods are in charge of the following activities:

- Bundling of incoming entities in the System Module with production Kanban: as soon as a Kanban is available, it is attached to the entity and the bundle is sent downward.

- Detachment of Kanban: according to the implemented control policy, Module_Kanban manages the detachment of Kanban.

- Collection of spare Kanban: once Kanban are freed, they get sent back to their original Module_Kanban and start circulating again.

- Management of rework processes and scrap: if an entity requires to be re-processed, Module_Kanban controls its access to the process according to Kanban availability.

- Demand Kanban control: in Presence of Demand policies, if a station is specified by the user as the demand decoupling point, demand Kanban are sent to the station according to customers' demand rate. Entities are processed only if a customer requires it. This mechanism works together with components' final warehouses, which forward customers' demands to the proper decoupling points (Fig 16).

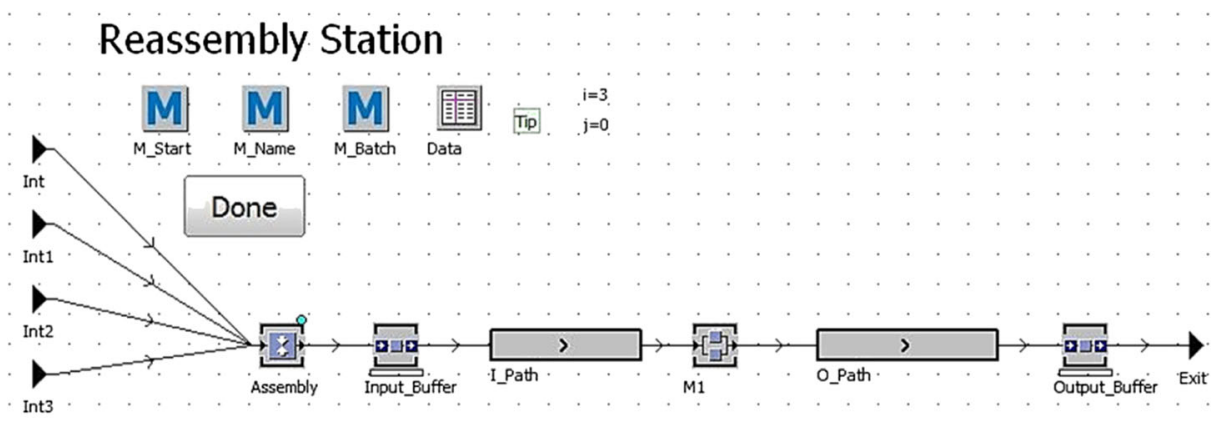

Fig. 14 Reassembly Station 


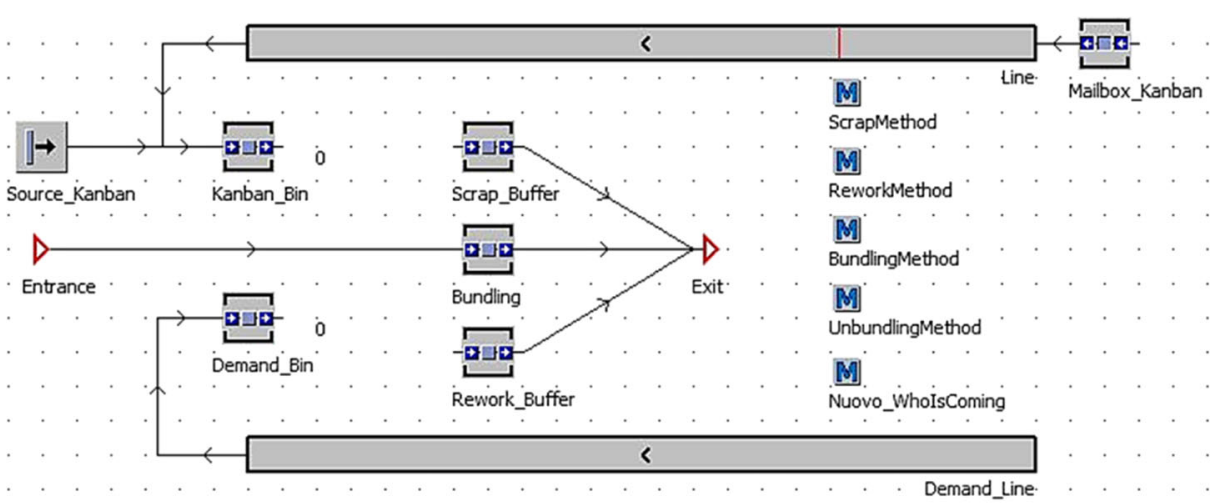

Fig. 15 Module_Kanban insight

The selection of the control policy made by the user triggers the entire tuning of the model. In fact, Module_Kanban dynamically handles material flows according to the central settings provided by system-level controlling method.

\section{Verification and validation}

Once the simulation framework is developed, the goal of the verification is to check the formal correctness of the model. This step has been accomplished through the simulator debugger, the usage of software animations and the monitoring of the simulator's events list.

Following the verification phase, the model validation was performed to verify the reliability of the model. It has been compared to existing performance evaluation methods:

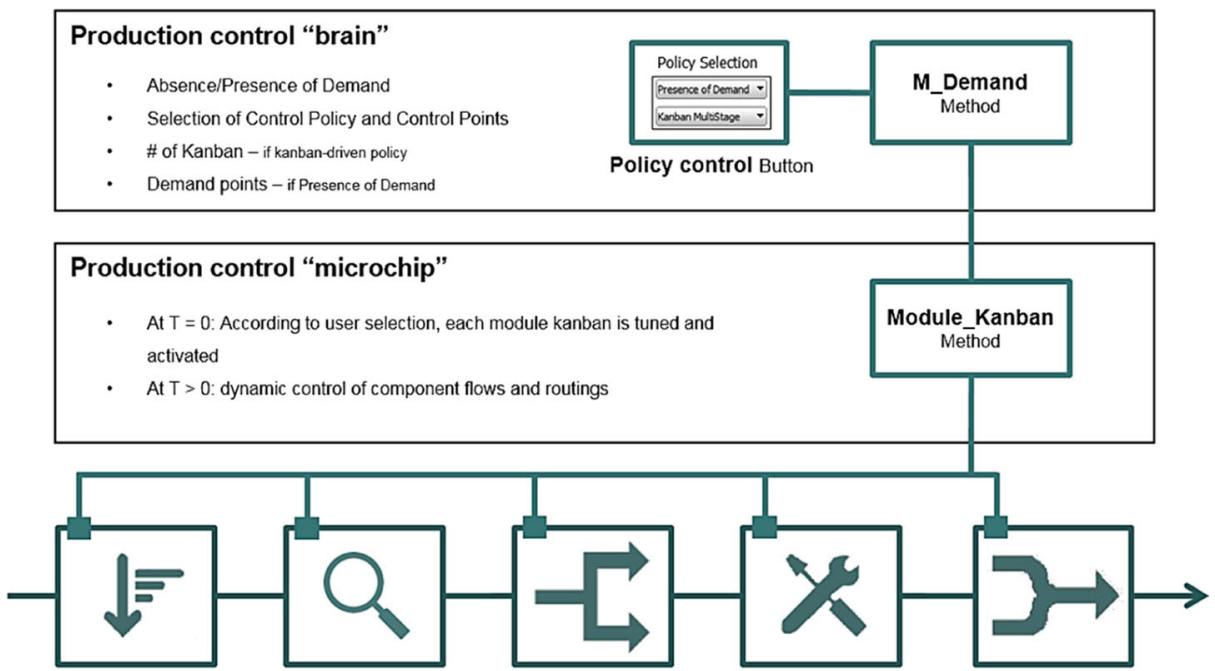

Fig. 16 Multi-level material flow control 
precisely, with a Markov Chain based method and Queuing Network. For each peer comparison, the questioned models must be aligned in terms of functioning hypothesis.

The figure below reports the validation process performed over a semi-open Jackson Network. This model is solvable using a Convolution algorithm and enables to represent a ConWIP system, thanks to the introduction of a fictitious resource Node0, which transform the network from an open one into a closed one. Since the mechanism behind the functioning of the Module_Kanban is the same throughout the different implemented policies, the validation of the ConWIP configuration was enough to assert that the management of Kanban flow properly performs over all the token-based configurations.

The validation entailed the construction of the two equivalent systems shown in Fig. 17, evaluated over their expected throughput. Precisely, after proving the Gaussian distribution of the values from the simulation runs, a hypothesis test over the throughput equivalence has been performed. Results conclude that the tested features can be considered validated (Fig 18).

\section{Application to a real case study}

The developed model has been deployed for supporting performances forecasting of an independent Chinese remanufacturer, aiming at improving the operations through the application of production control policies. The product under analysis is a turbocharger made of eight target components. The process is the following: cores are collected, inspected and disassembled; then, each liberated target component follows its own process, made of cleaning, inspection and reconditioning, until it reaches the reassembly phase, which precedes the final testing and packaging one. The company faces problems due to input materials uncertainty and processes variability. In the first case, in spite of the abundance of cores, the arrival pace is not predictable enough to grant a smooth flow. In the second, given that remanufacturing processes are characterized by several manual activities - whose time often
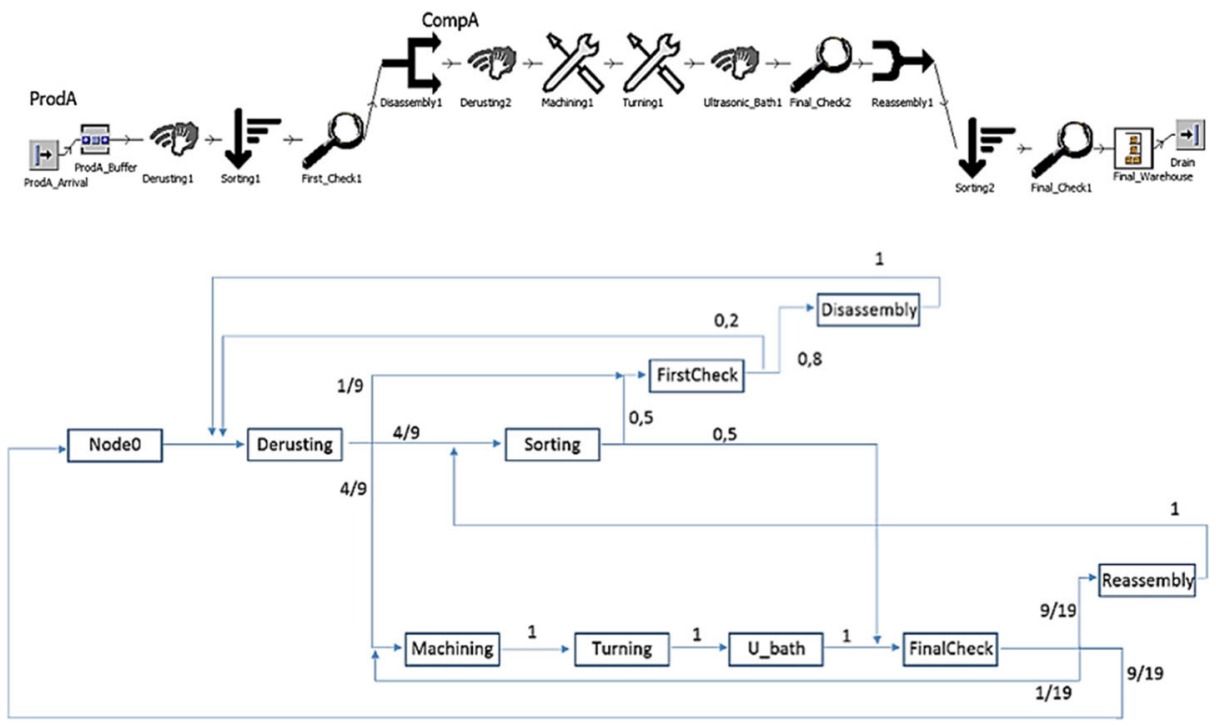

Fig. 17 Simulation model vs Jackson Network 
depends on the product's quality - the higher the instability of such parameter is, the wider is the distributions of the processing times are.

Using the data from the Value Stream Mapping (VSM) of the case under analysis, a process has been modelled through the developed simulation tool, as represented in Fig. 19.

Objective of the case has been to provide the customer with a sensitivity analysis of the average throughput time under the variation of the deployed token-based control policy. The evaluation is performed considering two different time references, related to distinct systems, which differ depending on the inclusion or not of the initial buffer, i.e. the one after the Source. The first, the Throughput Time, takes into account the time spent by cores from the initial buffer on, while the second, the Process Time, only considers the average time spent once the core outgoes on that buffer and enters the first inspection. The reason behind this choice is that the control policies have a direct impact only on the portion of system below the initial buffer, whereas the amount of parts stored there - hence the time spent - is not directly controllable, being also a consequence of the arrivals distributions.

All the four token-based control policies have been applied in simulation and, each policy has been evaluated both in Absence and Presence of Demand situation. In the last case, the only demand point set has been the initial inspection process. On the contrary, in both cases, the selection of control points and the respective number of Kanban to be managed has been carried out heuristically, in order to streamline the material flow. Such goal is achievable considering the technological routing of each component and the respective sojourn time. Afterward, the resources utilization and batch sizing policy have been considered for fine-tuning the decisions.

The simulation experiments excluded the data gathered during the ramp up time, which amount has been computed according to the methodology of Heidelberger and Welch [27]. Each experiment, performed once per policy type, has been set of thirty runs, to guarantee the normality of output data according to the central limit theorem [28]. Finally, the company, according to its needs, has defined the runs' length.

The implementation of production policies was effective over the Process Time reduction: as shown in Fig. 20, all the token-based policies improved the process time both in average values and in related standard deviation. Of course, performances variation between policies is due to the intrinsic nature of policies themselves. However, the choice of the appropriate solution depends on the targets and constraints set by the company. For instance, if the objective parameter is the average Process Time, then the best solution is the Multi-Stage control, in presence of demand. Differently, in case a stable Process Time is required, an Echelon control, also in presence of demand would be suggested. In general, control policies performed better in combination with the demand-pull mechanism: this is due to less unrequired material that enters the system, avoiding its overloading and making the overall process leaner.

\begin{tabular}{|l|r|r|r|r|r|r|r|}
\hline Clients & 10 & 15 & 20 & 25 & 30 & 40 & 50 \\
\hline Observations & 30 & 30 & 30 & 30 & 30 & 30 & 30 \\
\hline Expected Th & 2092,15 & 2448,67 & 2664,69 & 2804,01 & 2897,82 & 3009,65 & 3069,10 \\
\hline Actual TH & 2092,07 & 2448,73 & 2665,23 & 2803,93 & 2897,23 & 3008,77 & 3068,7 \\
\hline Error & $0,0042 \%$ & $-0,0026 \%$ & $-0,0205 \%$ & $0,0027 \%$ & $0,0201 \%$ & $0,0294 \%$ & $0,0131 \%$ \\
\hline Actual StDev & & & & & & & \\
\hline$z(\alpha / 2)=z(0,975)$ & 2,56 & 3,29 & 4,32 & 6,18 & 6,09 & 9,19 & 9,72 \\
\hline Lower Bound Th & 2087,06 & 2442,29 & 2656,77 & 2791,82 & 2885,30 & 2990,76 & 3049,64 \\
\hline Upper Bound Th & 2097,07 & 2455,17 & 2673,70 & 2816,05 & 2909,16 & 3026,77 & 3087,76 \\
\hline
\end{tabular}

Fig. 18 Validation results 


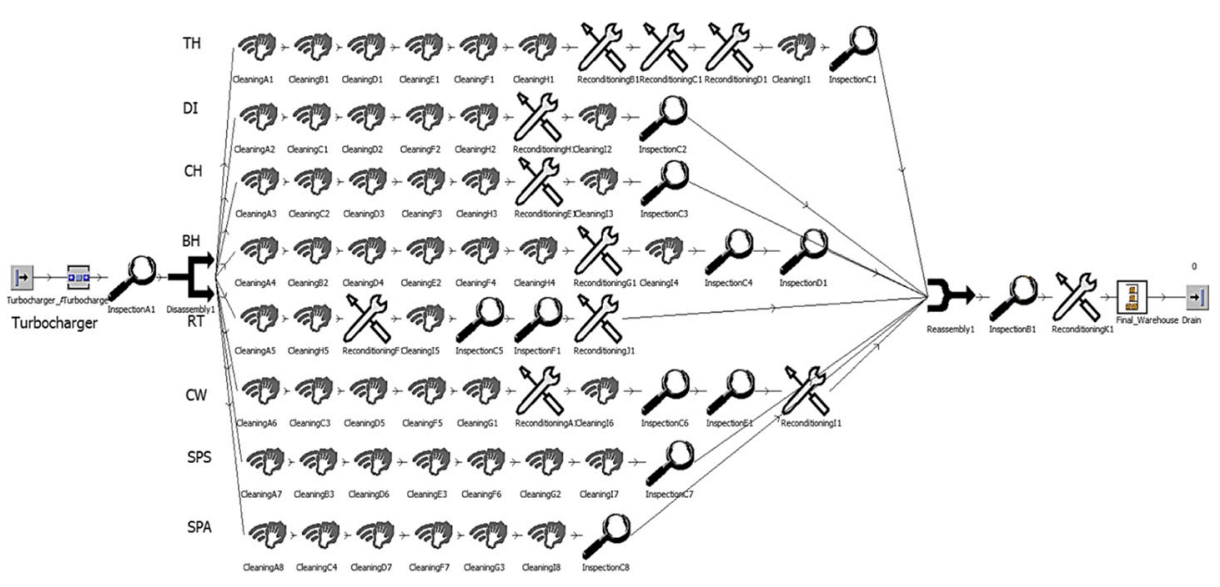

Fig. 19 Turbocharger model

Regardless of the obtained results, it is worth mentioning that the cooperation of modularization, product-oriented simulation and flow control at module level, through Module_Kanban, has represented an effective solution for the management of different production control policies. Additionally, the modular approach sharply grasps high level of descriptive detail thanks to the coding complexity embedded in the model. Such accuracy is expressed by the wide KPIs overview provided by simulation experiments.

\section{Conclusions and Outlook}

In this work - differently from previous researchers - the simulation model has not been used to validate thesis about the application of production-control policies in remanufacturing. Reversely, having seen the goodness of their application, a simulation model able to handle them in detail and available to support customized analysis, has been developed.

Downward the design, development, validation and application of the model, it is possible to assert that the cooperation of modularization, product-oriented architecture and flow control at module level - through Module_Kanban - represents, for remanufacturing systems, an effective solution for the management of different production control policies. This was achieved due to the inclusion inside the System Modules of the Module_Kanban; the Module_Kanban, in fact, is connected on one side to the system level - represented from the module M_Demand - while on the other side it is rooted inside each product operation, allowing a tight and precise control on them. Additionally, the modular approach has been useful to keep the representation simple, despite the great modelling complexity rooted within and across System Modules, enabling the model to attain a high rate of descriptive detail, both at system and station level. An even broader

\begin{tabular}{|c|c|c|c|c|c|c|c|c|c|c|}
\hline & \multicolumn{2}{|c|}{ Baseline Case } & \multicolumn{2}{|c|}{ ConWIP } & \multicolumn{2}{|c|}{ Multi Stage } & \multicolumn{2}{|c|}{ Echelon } & \multicolumn{2}{|c|}{ Single Stage } \\
\hline & Abs of Dem & Pres of Dem & Abs of Dem & Pres of Dem & Abs of Dem & Pres of Dem & Abs of Dem & Pres of Dem & Abs of Dem & Pres of Dem \\
\hline Avg Th Time $\sigma[\mathrm{min}, \mathrm{sec}]$ & 390,99 & 439,24 & 411,67 & 452,42 & 385,25 & 417,54 & 398,37 & 424,26 & 396,05 & 482,80 \\
\hline$\triangle$ Avg Th Time $\sigma$ (over Baseline) & & & $-5,29 \%$ & $-3,00 \%$ & $1,47 \%$ & $4,94 \%$ & $-1,89 \%$ & $3,41 \%$ & $-1,29 \%$ & $-9,92 \%$ \\
\hline Avg Process Time $[\mathrm{min}, \mathrm{sec}]$ & $2.869,01$ & $2.491,04$ & $2.573,41$ & $2.462,75$ & $2.234,89$ & $2.230,65$ & $2.508,03$ & $2.387,79$ & $2,437,52$ & $2.424,69$ \\
\hline Avg Process Time $\sigma[\mathrm{min}, \mathrm{sec}]$ & 390,99 & 266,63 & 269,38 & 252,06 & 271,15 & 249,05 & 217,72 & 213,11 & 226,55 & 222,16 \\
\hline
\end{tabular}

Fig. 20 Case study results 
level of system description could be achieved taking into account the environmental performances that are tailored over remanufacturing activities [29].

Going into the margin for further researches, the feeling acquired throughout the work is that Remanufacturing still sees a lack of knowledge when it comes to merging the aspects related to business modelling and the operational field. It would be interesting to understand how decisions at a former level, for example concerning the kind of contracts for the return of cores, could be matched with decisions at operational level. Among which, as seen, the production-control policies.

\section{References}

1. Opresnik D, Taisch M (2015) The manufacturer's value chain as a service - the case of remanufacturing. J Remanuf 5(1):2. doi:10.1186/s13243-015-0011-x

2. Colledani M, Copani G, Tolio T (2014) De-manufacturing Systems. Variety Manag Manuf 17:14-19

3. Butzer S, Schötz S, Kruse A, Steinhilper R (2014) Managing complexity in remanufacturing focusing on production organisation. In: Zaeh M (ed) Enabling manufacturing competitiveness and economic sustainability: proceedings of the 5th international conference on changeable, agile, reconfigurable and virtual production (CARV 2013). Springer, Munich, pp 365-370

4. Guide VDR (1996) Scheduling using drum-buffer-rope in a remanufacturing environment. Int J Prod Res 34(4):1081-1091

5. Guide VDR, Kraus ME, Srivastava R (1997) Scheduling policies for remanufacturing. Int J Prod Econ 48(2): 187-204

6. Souza GC, Ketzenberg ME (2002) Two-stage make-to-order remanufacturing with service-level constraints. Int J Prod Res 40(2):477-493

7. Souza GC, Ketzenberg ME, Guide VDR (2002) Capacited Remanufacturing with service level constraints. Prod Oper Manag 11(2):231-248

8. Zhang R, Ong SK, Nee AYC (2015) A simulation-based genetic algorithm approach for remanufacturing process planning and scheduling. Appl Soft Comput 37:521-532

9. Gershenson JK, Prasad GJ (1997) Modularity in product design for manufacturability. Int J Agil Manuf 1(1):99-110

10. Miller TD, Elgard P (1998) Defining modules, modularity and modularisation, Design for Integration in Manufacturing, Proceedings of the 13th IPS Research Seminar, Fugsloe, Aalborg University

11. Smith JS, Valenzuela JF Mukkamala PS (2003) Designing reusable simulation modules for electronics manufacturing systems Proceedings of the 2003 Winter Simulation Conference 2:1281-1289

12. Martínez FM (2002) Bedia, Luis Miguel Arreche: Modular simulation tool for modelling JIT manufacturing. Int J Prod Res 40(7):1529-1547

13. Lee HY, Choi BK (2011) Using workflow for reconfigurable simulation-based planning and scheduling system. Int J Comput Integr Manuf 24(2):171-187

14. Tang O, Teunter R (2006) Economic lot scheduling problem with returns. Prod Oper Manag 15(4):488-497

15. Veerakamolmal P, Gupta SM (1998) High-mix/low-volume batch of electronic equipment disassembly. Comput Ind Eng 35(1-2):65-68

16. Gupta SM, Al-Turki YAY (1997) An algorithm to dynamically adjust the number of kanbans in stochastic processing times and variable demand environment. Prod Plan Control 8(2):133-141

17. Korugan A, Gupta SM (2014) An adaptive CONWIP mechanism for hybrid production systems. Int J Adv Manuf Technol 74(5-8):715-727

18. Teunter R, Kaparis K, Tang O (2008) Multi-product economic lot scheduling problem with separate production lines for manufacturing and remanufacturing. Eur J Oper Res 191(3):1241-1253

19. Teunter R, Tang O, Kaparis K (2009) Heuristics for the economic lot scheduling problem with returns. Int J Prod Econ 118(1):323-330

20. Guide VDR Jr, Srivastava R (1997) Buffering from material recovery uncertainty in a recoverable manufacturing environment. J Oper Res Soc 48(5):519-529

21. Guide V, Daniel R, Srivastava R (1997) An evaluation of order release strategies in a remanufacturing environment. Comput Oper Res 24(1):37-47

22. Guide V, Daniel R, Souza GC, van der Laan E (2005) Performance of static priority rules for shared facilities in a remanufacturing shop with disassembly and reassembly. Eur J Oper Res 164(2):341-353

23. Banks J, Carson II J, Nelson B, Nicol D (2010) Discrete-event system simulation, 5th edn. Pearson 
24. Liberopoulos, G. (2013) Production Release Control: Paced, WIP-Based or Demand-Driven? Revisiting the Push/Pull and Make-to-Order/Make-to-Stock Distinctions. In: Smith J., Tan B. (eds) Handbook of Stochastic Models and Analysis of Manufacturing System Operations. International Series in Operations Research \& Management Science, vol 192. Springer, New York, NY

25. Steinhilper R (1998) Remanufacturing: the ultimate form of recycling. Fraunhofer IRB Verlag, Stuttgart

26. Hopp WJ, Spearman ML (2011) Factory physics. Waveland Press. GS link: https://scholar.google. be/scholar?q=Hopp+WJ,+Spearman+ML+(2011)+Factory+physics\&hl=it\&as_sdt=0\&as_vis=1\&oi= scholart\&sa=X\&ved=0ahUKEwjmn7e-m6rVAhUQU1AKHfrLBccQgQMIJzAA

27. Heidelberger P, Welch PD (1983) Simulation run length control in the presence of an initial transient. Oper Res 31(6):1109-1144

28. Ross SM (2009) Introduction to Probability and Statistics for Engineers and Scientists, 4th edn. Elsevier Academic Press. GS link: https://books.google.it/books?hl=it\&lr=\&id=BaPOv33uZCMC\&oi=fnd\&pg= PP1\&dq=Introduction+to+Probability+and+Statistics+for+Engineers+and+Scientists \&ots=C 9 NKuVtlwU\&sig=vUSmqKEQyn4WevUBDkb2yefdKjE\%23v=onepage\&q=Introduction\%20to\%20 Probability $\% 20$ and $\% 20$ Statistics $\% 20$ for $\% 20$ Engineers $\% 20$ and $\% 20$ Scientists $\&=$ false

29. Graham I, Goodall P, Peng Y, Palmer C, West A, Conway P, Mascolo JE, Dettmer FU (2015) Performance measurement and KPIs for remanufacturing. J Remanuf 5(1):10 